\title{
Deletion of the $m m p L 4 b$ gene in the Mycobacterium abscessus glycopeptidolipid biosynthetic pathway results in loss of surface colonization capability, but enhanced ability to replicate in human macrophages and stimulate their innate immune response
}

\author{
Rachid Nessar, ${ }^{1}$ Jean-Marc Reyrat, ${ }^{1} \dagger$ Lisa B. Davidson ${ }^{2}$ \\ and Thomas F. Byrd ${ }^{2,3}$ \\ ${ }^{1}$ Inserm-U 1002, Groupe Avenir, Université Paris V-Descartes, Faculté de Médecine, Site Necker, \\ F-75730 Paris Cedex 15, France \\ ${ }^{2}$ Department of Medicine, New Mexico Veterans Health Care System, 1501 San Pedro SE, \\ Albuquerque, NM 87108, USA \\ ${ }^{3}$ The University of New Mexico School of Medicine, Albuquerque, NM 87108, USA
}

Correspondence

Thomas F. Byrd

tbyrd@salud.unm.edu

Received 1 November 2010

Revised 18 January 2011

Accepted 27 January 2011

\begin{abstract}
Mycobacterium abscessus is considered to be the most virulent of the rapidly growing mycobacteria. Generation of bacterial gene knockout mutants has been a useful tool for studying factors that contribute to virulence of pathogenic bacteria. Until recently, the optimal genetic approach to generation of $M$. abscessus gene knockout mutants was not clear. Based on the recent identification of genetic recombineering as the preferred approach, a $M$. abscessus mutant was generated in which the gene $m m p L 4 b$, critical to glycopeptidolipid synthesis, was deleted. Compared to the previously well-characterized parental strain $390 \mathrm{~S}$, the $\mathrm{mmp} L 4 B$ deletion mutant had lost sliding motility and the ability to form biofilm, but acquired the ability to replicate in human macrophages and stimulate macrophage Toll-like receptor 2 . This study demonstrates that deletion of a gene associated with expression of a cell-wall lipid can result in acquisition of an immunostimulatory, invasive bacterial phenotype and has important implications for the study of $M$. abscessus pathogenesis at the cellular level.
\end{abstract}

\section{INTRODUCTION}

Mycobacterium abscessus is a rapidly growing non-tuberculous mycobacterium (NTM) which causes skin and soft tissue infections, and is an important cause of pneumonia in patients with structural abnormalities of the lung (De Groote \& Huitt, 2006; Howard \& Byrd, 2000; Griffith et al., 1993). It is also a pulmonary pathogen in patients with cystic fibrosis (Cullen et al., 2000; Fauroux et al., 1997; Olivier et al., 2003; Sermet-Gaudelus et al., 2003). In some instances, isolation of M. abscessus from the sputum is not

†Deceased 28 October 2009.

Abbreviations: GPL, glycopeptidolipid; MBC, mimimum bactericidal concentration; NHS, normal human serum; NTM, non-tuberculous mycobacterium/mycobacteria; MDM, monocyte-derived macrophage; PIM, phosphatidyl-myo-inositol mannoside; TLR2, Toll-like receptor 2; TNF, tumour necrosis factor.

Three supplementary tables are available with the online version of this paper. associated with progressive lung infection and may be the result of transient airway colonization (Griffith et al., 2007; Jönsson et al., 2007). In other clinical situations, lung infection is progressive and refractory to antibiotic therapy, necessitating lung resection for cure (Griffith et al., 1993; Daley \& Griffith, 2002; Sherwood et al., 2005). This latter clinical scenario has resulted in the view that M. abscessus is the most virulent of the rapidly growing mycobacteria that cause invasive lung infection (Petrini, 2006).

One of our laboratories has focused their studies on three well-characterized isogenic M. abscessus variants; 390R (rough colony morphology), 390S (smooth colony morphology) and 390V (rough colony morphology). $M$. abscessus 390R, a patient isolate, spontaneously dissociated to the $390 \mathrm{~S}$ variant when subcultured (Byrd \& Lyons, 1999). 390V, an isogenic rough revertant, then arose from 390 S on subculture (Howard et al., 2006). All three variants have stable phenotypes with low rates of spontaneous reversion (Byrd \& Lyons, 1999; Howard et al., 2006). We 
have demonstrated that the smooth colony phenotype of $M$. abscessus is due to expression of glycopeptidolipid (GPL) in the bacterial cell wall; GPL is minimally expressed by rough variants (Howard et al., 2006). We have also demonstrated that the rough variants $390 \mathrm{R}$ and $390 \mathrm{~V}$ are able to grow in human macrophages and stimulate macrophage Toll-like receptor 2 (TLR2), while the 390S variant lacks these capabilities (Byrd \& Lyons, 1999; Howard et al., 2006; Rhoades et al., 2009). Unlike the rough variants, the $390 \mathrm{~S}$ variant is able to form biofilms and exhibits sliding motility. We have postulated that this is due to GPL expression by 390S, which is supported by genetic studies demonstrating that GPL is necessary for both biofilm formation and sliding motility in Mycobacterium smegmatis, a non-pathogenic NTM (Recht et al., 2000). We have provided evidence that expression of GPL facilitates the M. abscessus colonization phenotype, but 'masks' underlying bioactive cell-wall lipids involved in virulence (Rhoades et al., 2009). In this study we have used genetic recombineering to delete the M. abscessus $390 \mathrm{~S}$ $m m p L 4 b$ gene, a gene coding for a membrane protein which has been found to play an essential role in GPL expression by NTM (Recht et al., 2000; Ripoll et al., 2007; Medjahed \& Reyrat, 2009). Using this deletion mutant we provide direct evidence that GPL plays a role in both the colonization and invasion phenotypes of $M$. abscessus.

\section{METHODS}

Mycobacterial strains and preparation of bacterial stocks. Strains used in the present study are listed in Supplementary Table S1, available with the online version of this paper. The M. abscessus $390 \mathrm{~S}$ smooth colony variant was used as wild-type in these studies (Byrd \& Lyons, 1999; Howard et al., 2002, 2006; Rhoades et al., 2009). Bacteria were maintained as titrated frozen stocks stored at $-70{ }^{\circ} \mathrm{C}$ with intermittent passage for 3 days on Middlebrook $7 \mathrm{H} 11$ agar plates supplemented with Middlebrook OADC (BD) followed by flash freezing. To prepare single-cell frozen bacterial stocks for experiments, lawns of the different bacterial strains were plated on Middlebrook 7H11 OADC agar plates and incubated at $37^{\circ} \mathrm{C}$. After 3 days bacteria were harvested and placed into sterile Eppendorf tubes containing $1.0 \mathrm{ml}$ sterile PBS and five glass beads. Tubes were pulse-vortexed 50 times, after which residual aggregates of bacteria were allowed to settle for $20 \mathrm{~min}$. The top $500 \mu \mathrm{l}$ of bacterial supernate was removed and the supernates from two to four tubes were pooled in $50 \mathrm{ml}$ conical tubes. Bacteria were then sonicated on high power in a sonicating waterbath for $20 \mathrm{~s}$ to break apart any residual bacterial aggregates. The bacterial suspension was aliquoted into multiple Eppendorf tubes, which were then flash frozen and stored at $-70{ }^{\circ} \mathrm{C}$. Individual tubes were thawed and titrated to determine c.f.u. for a particular frozen stock.

Construction of the $\boldsymbol{M}$. abscessus $\mathbf{m m p L 4 b}$ mutant. M. abscessus $390 \mathrm{~S}$ is a well-characterized smooth variant which expresses abundant GPL (Byrd \& Lyons, 1999; Howard et al., 2002, 2006; Rhoades et al., 2009). The $m m p L 4 b$ gene is a non-essential gene which is necessary for M. abscessus GPL production. In a prior study, this gene was used as a target to compare three different approaches for generating allelic-exchange mutants in $M$. abscessus. It was found that the recombineering system was the most efficient method for this purpose (Medjahed \& Reyrat, 2009), and thus this was the approach used in our study. The plasmids and primers used are listed in
Supplementary Tables S2 and S3, respectively. The marked mutation construct of the mmpL4b gene (MAB_4115c) was generated by PCR. Primers $m m p L 4 b 1-\mathrm{F}$ and $m m p L 4 b 1-\mathrm{Rv}$, containing EcoRI and SalI sites, respectively, were used to amplify a fragment (fragment 1) encoding N-terminal amino acids $1-3$, along with a 908 bp sequence upstream of the $m m p L 4 b$ gene. Similarly, primers $m m p L 4 b 2-\mathrm{F}$ and mmpL4b2-Rv, containing SalI and ApaI sites, respectively, were used to amplify a fragment (fragment 2) encoding amino acids 985-987, along with a $950 \mathrm{bp}$ region downstream of the $m m p L 4 b$ gene. Fragments 1 and 2 were digested by either EcoRI/SalI or SalI/ApaI (Biolabs) and cloned in EcoRI-ApaI-digested pBluescript II SK(+) (Stratagene) to generate pBSK- $\Delta m m p L 4 b$. The zeocin resistance cassette was amplified from pLYG204 Zeo (Howard et al., 1995) using primers Zeo-F and Zeo-Rv, purified and digested by SalI and cloned into the SalI site of pBSK- $\Delta m m p L 4 b$ to generate pBSK$\Delta m m p L 4 b::$ Zeo. This allelic-exchange substrate was used to perform mutagenesis in M. abscessus $390 \mathrm{~S}$.

Complementation of the $\boldsymbol{M}$. abscessus mmpL4b mutant. The complemented strain was constructed as follows. The $m m p L 4 b$ gene (MAB_4115c) with 300 bp upstream and downstream from the target gene was amplified by PCR using polymerase Pfu (Biolabs) and the primers $m m p L 4 b$-compF and $m m p L 4 b$-compRv, each containing a $X b a \mathrm{I}$ site (Supplementary Table S3). The genomic DNA of $M$. abscessus 390S was used as template. The PCR product was purified, digested with $\mathrm{XbaI}$ and cloned into plasmid pNBV1 (Howard et al., 1995), which was previously digested by XbaI, dephosphorylated by alkaline phosphatase and purified to generate pNBV1-mmpL4b. This construct was electroporated into the mutant strain $M$. abscessus $\Delta m m p L 4 b$ and the transformants were selected on hygromycin $\left(1000 \mu \mathrm{g} \mathrm{ml}^{-1}\right)$.

Sliding motility and biofilm formation. To assess sliding motility, a liquid culture of $M$. abscessus was dropped $(10 \mu \mathrm{l})$ onto plates containing $7 \mathrm{H} 9$ medium ( $0.3 \%$ agar $)$ without an added carbon source. Plates were incubated at $37^{\circ} \mathrm{C}$ and colony spread was assessed after 7 days.

To assess biofilm formation, bacteria were cultured in a Calgary biofilm device (MBEC Bioproducts) (Ceri et al., 1999) at a concentration of $2 \times 10^{4}$ c.f.u. in $200 \mu$ l Iscove's medium (Gibco) per well. The culture was incubated at $37{ }^{\circ} \mathrm{C}$ on a shaker set to 100 r.p.m. and the medium was replaced every $24 \mathrm{~h}$ for a total culture time of $72 \mathrm{~h}$. The biofilm pegs attached to the device lid were then placed in a new 96-well plate with $200 \mu$ l Iscove's medium in each well and left for $2 \mathrm{~min}$ as a wash step. Pegs were then removed from the lid (four pegs were harvested per bacterial strain), placed in tubes containing $1 \mathrm{ml}$ sterile PBS, and sonicated for 20 s. Bacterial c.f.u. were then titrated on $7 \mathrm{H} 11$ agar plates.

M. abscessus infection of human macrophages. Human mononuclear cells were isolated from buffy coats purchased from United Blood Services and cultured for $48 \mathrm{~h}$ in Teflon jars in Iscove's/ $5 \%$ NHS medium at $37{ }^{\circ} \mathrm{C}$ in $5 \% \mathrm{CO}_{2}$ to facilitate monocyte maturation. Human monocytes were isolated by adherence to tissue culture wells (Falcon) followed by incubation for $24 \mathrm{~h}$ at $37{ }^{\circ} \mathrm{C}$ in medium. Immediately prior to challenge with bacteria, monocytederived macrophage (MDM) monolayers were washed twice to remove non-adherent cells. MDM monolayers were then infected with $5.0 \times 10^{5} \mathrm{M}$. abscessus c.f.u. $\mathrm{ml}^{-1}$ in $0.5 \mathrm{ml}$ Iscove's $/ 5 \% \mathrm{NHS}$. At the time the infecting inocula of the different variants were added to wells, infecting inocula from the same stock tubes were also placed on 7H11 plates containing zeocin at a concentration of $50 \mu \mathrm{g} \mathrm{ml} \mathrm{m}^{-1}$. After 90 min incubation, the MDM monolayers were washed three times with $0.5 \mathrm{ml}$ Iscove's $/ 5 \%$ NHS to remove remaining extracellular bacteria, and $0.5 \mathrm{ml}$ Iscove's $/ 5 \%$ NHS added back to each well. Cell lysates containing bacteria were harvested at time 0 and 
c.f.u. plated as described previously (Howard et al., 2006). At $24 \mathrm{~h}$, MDM monolayers were washed twice with $0.5 \mathrm{ml}$ Iscove's $/ 5 \%$ NHS to remove nonadherent cells and extracellular bacteria. Cell lysates were plated and $0.5 \mathrm{ml}$ Iscove's $/ 5 \%$ NHS was added back to remaining wells. This was repeated at 48 and $72 \mathrm{~h}$, with a final plating of cell lysates at $72 \mathrm{~h}$. We have found that NHS is a necessary component of wash medium as it prevents non-specific adherence of $M$. abscessus GPL-expressing variants to tissue culture wells (Greendyke \& Byrd, 2008). Non-specific adherence of bacteria to tissue culture wells can lead to overgrowth of the bacteria in tissue culture media and spurious results. Progression of infection in MDM monolayers is dependent on $M$. abscessus spreading from initially infected macrophages to uninfected macrophages as occurs with intracellular bacterial pathogens such as Legionella pneumophila (Byrd \& Horwitz, 1989). Although M. abscessus can multiply to a limited extent in tissue culture medium, extensive washing of monolayers after initial infection and at time points throughout the experiment minimizes extracellular growth as an artefact (Greendyke \& Byrd, 2008). In prior studies we have demonstrated that after extensive washing of MDMs to reduce extracellular bacteria, the addition of amikacin to kill any residual extracellular bacteria does not change the outcome of macrophage infection comparing the $390 \mathrm{R}, 390 \mathrm{~V}$ and 390S M. abscessus variants (Howard et al., 2006; Greendyke \& Byrd, 2008). To assess whether addition of amikacin has an effect on the growth of the mmpL4b deletion mutant in MDMs we also compared washing alone to washing followed by the addition of amikacin for $6 \mathrm{~h}$ at a concentration of $64 \mu \mathrm{g} \mathrm{ml}^{-1}$, the minimum bactericidal concentration (MBC) for the 390 variants (Greendyke \& Byrd, 2008), prior to plating cell lysates for time 0 c.f.u. Middlebrook 7H9 broth (Difco) was used for dilution of cell lysates prior to plating for c.f.u. Middlebrook 7H11 agar (Difco) plates, with and without zeocin, were used for plating c.f.u. from infected monolayers. In addition, nuclear counts in replicate wells were determined, and c.f.u. standardized to $10^{5}$ nuclei to account for any differences in monocyte number in the different monolayers, as has been described previously (Byrd, 1997). Replicate monolayers treated in the same fashion were fixed with PBS/4\% paraformaldehyde and photographed with a Nikon TE1 $2000 \mathrm{U}$ phase-contrast microscope.

Induction of macrophage TNF $\alpha$ release by M. abscessus variants. Human monocytes were isolated, cultured in Teflon jars to generate MDMs, and plated in tissue culture wells as described above. After $24 \mathrm{~h}$ incubation in medium at $37{ }^{\circ} \mathrm{C}$ in $5 \% \mathrm{CO}_{2}$, the tissue culture wells containing MDM monolayers were washed three times with $0.5 \mathrm{ml}$ Iscove's medium, and $0.5 \mathrm{ml}$ Iscove's medium without NHS was added back to each well. Mouse anti-human TLR2 (eBioscience clone T2.5) $\left(10 \mu \mathrm{g} \mathrm{ml}^{-1}\right)$ and mouse $\mathrm{IgG}_{1}$ isotype control (eBioscience) $\left(10 \mu \mathrm{g} \mathrm{ml}^{-1}\right)$ were added to appropriate wells and incubated for $1 \mathrm{~h}$ at $37{ }^{\circ} \mathrm{C}$ in $5 \% \mathrm{CO}_{2}$. Wells were then infected with $2.5 \times 10^{6} \mathrm{M}$. abscessus c.f.u. per well (bacteria to macrophage ratio in the range of 10 to 1 ), and incubated at $37{ }^{\circ} \mathrm{C}, 5 \% \mathrm{CO}_{2}$ for $2 \mathrm{~h}$. Supernates were filtered through $0.22 \mu \mathrm{m}$ filter units and stored at $-70{ }^{\circ} \mathrm{C}$ for later determination of TNF $\alpha$ levels. TNF $\alpha$ levels were measured using the TNF $\alpha$ ELISA BD OptEIA (BD Biosciences). In addition, nuclear counts in replicate wells were determined at each time point, and TNF $\alpha$ was standardized to $10^{5}$ nuclei to account for any differences in macrophage number in monolayers subjected to different experimental treatments or conditions (Rhoades et al., 2009). We have found that this is critical for meaningful interpretation of results since it allows for standardization among different experimental groups. In addition, there is substantial variation in tissue culture adherence of MDMs from different donors; thus this also allows for meaningful comparisons between experiments.

GPL extraction and analysis. M. abscessus lipids were extracted from plate-grown cultures as previously described (Howard et al.,
2006). Briefly, cultures were extracted with $\mathrm{CHCl}_{3} / \mathrm{CH}_{3} \mathrm{OH}[2: 1, \mathrm{v} / \mathrm{v}$; $10 \mathrm{ml}$ (g wet weight bacteria) $\left.{ }^{-1}\right]$. Bacteria were extracted twice at $56{ }^{\circ} \mathrm{C}$ (15 min with sonication) and once at $4{ }^{\circ} \mathrm{C}$ overnight, and insoluble material was removed by centrifugation and filtration (0.2 $\mu \mathrm{m}$ PTFE filter; Millipore). Combined extracts were subjected to biphasic partitioning in $\mathrm{CHCl}_{3} / \mathrm{CH}_{3} \mathrm{OH} / \mathrm{H}_{2} \mathrm{O}(4: 2: 1$, by vol.). Lipids in the organic phase were stored at $-20{ }^{\circ} \mathrm{C}$. Total lipid extracts were resuspended at $10 \mu \mathrm{g} \mathrm{ml}^{-1}$ in $\mathrm{CHCl}_{3} / \mathrm{CH}_{3} \mathrm{OH}$ and spotted onto aluminium-backed silica gel-60 TLC plates (EM Science). Lipids were resolved in $\mathrm{CHCl}_{3} / \mathrm{CH}_{3} \mathrm{OH} / \mathrm{H}_{2} \mathrm{O}$ (100:14:0.8, by vol.). Plates were sprayed with 1-naphthol (Sigma; $3 \%$ with $10 \% \mathrm{H}_{2} \mathrm{SO}_{4}$ in $\mathrm{CH}_{3} \mathrm{OH}$ ) to identify characteristic GPL spots associated with M. abscessus $390 \mathrm{~S}$ (Howard et al., 2006).

Statistics. Data were compared using Student's $t$ test. Differences were considered significant with $P<0.05$.

\section{RESULTS}

\section{Generation of a M. abscessus $390 \mathrm{~S}$ mmpL4b deletion mutant}

To further define the role of GPL in M. abscessus pathogenesis, we used genetic recombineering to generate a deletion mutant deficient in GPL synthesis from our $390 \mathrm{~S}$ variant. We targeted the $m m p L 4 b$ gene, a non-essential gene that plays a key role in GPL synthesis and which has previously been demonstrated to be a suitable target for generating a $M$. abscessus GPL-deficient mutant (Medjahed \& Reyrat, 2009). After bacterial transformation and plating, a M. abscessus 390 S derivative colony with the rough phenotype was picked for further analysis. To confirm loss of $m m p L 4 b$, PCR was performed using primer sets flanking $m m p L 4 b 3-\mathrm{F}$ and $m m p L 4 b 3-R$ (Fig. 1) (Supplementary Table S3). Wild-type 390S yielded a $2960 \mathrm{bp}$ product, while the rough variant yielded a $560 \mathrm{bp}$ product consistent with loss of the mmpL $4 b$ gene. In addition, a Southern blot hybridized with a DNA probe overlapping the $m m p L 4 b$ gene generated different banding patterns comparing wild-type $390 \mathrm{~S}$ and the rough variant (Fig. 2). Consistent with the rough phenotype (Fig. 3), these results confirm loss of mmpL $4 b$ in the transformant. We have previously characterized GPL expression of wild-type $390 \mathrm{~S}$ by TLC (Howard et al., 2006). TLC analysis of wild-type $390 \mathrm{~S}$ demonstrated characteristic GPL spots while $390 \mathrm{~S}$ $\Delta m m p L 4 b$ demonstrated minimal GPL expression, consistent with loss of mmpL $4 b$ (Fig. 3). A complemented strain was generated by incorporating the construct pNBV1-mmpL4b, carrying 300 bp upstream and downstream of the mmpL4b gene, into the M. abscessus $390 \mathrm{~S}$ $\Delta m m p L 4 b$ mutant. This variant exhibited the smooth phenotype and expressed GPL (Fig. 3).

\section{S $\Delta m m p L 4 b$ has lost sliding motility}

Both sliding motility and biofilm formation are believed to facilitate survival of NTM as environmental saprophytes. GPL in M. smegmatis has been demonstrated to be essential for sliding motility and biofilm formation (Recht et al., 
(a)

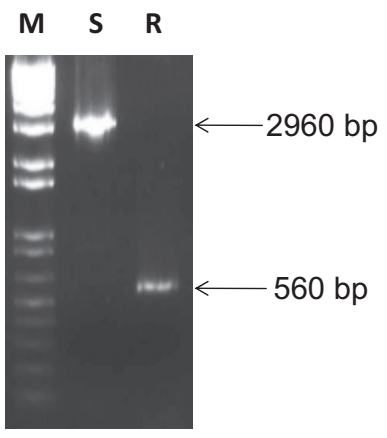

(b)

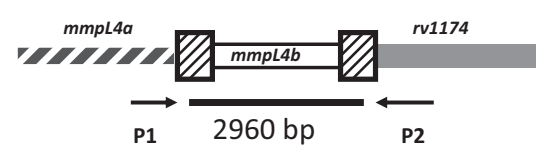

(S)

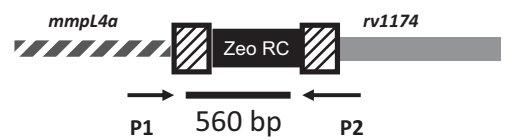

Fig. 1. (a) PCR analysis of the $m m p L 4 b$ locus in M. abscessus 390 S wild-type (lane $S$ ) and the $m m p L 4 b$ knockout strain (lane R). Lane M, size markers. (b) Schematic representation of the wild-type $m m p L 4 b$ locus and the allelicexchange mutant with the zeocin resistance cassette (Zeo RC). The predicted phenotype is indicated in parentheses: S, smooth; R, rough.
2000). In addition, the presence of GPL in M. abscessus has been correlated with the ability of different variants to form biofilm and exhibit sliding motility (Howard et al., 2006). Unlike wild-type 390 S and the complemented strain, 390S $\Delta m m p L 4 b$ did not exhibit sliding motility or biofilm formation (Fig. 4a, b). M. abscessus $390 \mathrm{~V}$ is a naturally occurring spontaneous mutant derived from 390S which has lost the ability to express GPL, and is thus an ideal comparator strain for $390 \mathrm{~S} \Delta m m p L 4 b$. When compared in the same experiments for biofilm formation and sliding motility, $390 \mathrm{~V}$ and $390 \mathrm{~S} \Delta m m p L 4 b$ did not form biofilms or exhibit sliding whereas wild-type $390 \mathrm{~S}$ did so (Fig. 4c and not shown). These results conclusively demonstrate a critical role for GPL in M. abscessus sliding motility and biofilm formation.

\section{$390 \mathrm{~S} \Delta m p L 4 b$ has acquired the ability to stimulate human macrophage TLR2}

We have previously demonstrated that our rough $M$. abscessus variants lacking GPL (390R and 390V) stimulate the human macrophage innate immune response through TLR2 stimulation. At least one class of the M. abscessus cell-wall ligands mediating this interaction are the phosphatidyl-myo-inositol mannosides (PIMs). Although the intact $390 \mathrm{~S}$ variant does not stimulate TLR2 signalling in human macrophages, $390 \mathrm{~S}$ from which GPL has been selectively removed acquires the ability to stimulate TLR2. Since 390S, 390R and 390V all possess similar PIMs in their cell walls capable of stimulating TLR2, this led to the conclusion that GPL is masking underlying PIMs in the $M$. abscessus $390 \mathrm{~S}$ cell wall, preventing their interaction with human macrophage TLR2 (Rhoades et al., 2009).

We tested the ability of the 390S $\Delta m m p L 4 b$ deletion mutant to stimulate human macrophage TLR2. We have previously demonstrated the specificity of the M. abscessusTLR2 interaction in experiments using HEK cells transfected with TLR2, and in TLR2-blocking antibody experiments (Rhoades et al., 2009). Here we demonstrate that $390 \mathrm{~S} \Delta m m p L 4 b$ acquires the ability to stimulate human macrophage TLR2 to a similar extent as our comparator strain 390V (Fig. 5). These results, taken with our previous study (Rhoades et al., 2009), demonstrate that GPL is masking underlying $M$. abscessus 390 S ligands that bind to the human macrophage TLR2 receptor.

\section{$390 S \Delta m m p L 4 b$ has acquired the ability to replicate in human macrophages}

The rough $M$. abscessus variants we have characterized which lack GPL (390R and 390V) are able to replicate in human macrophages, but M. abscessus 390S is not. Our data indicate that GPL masks underlying bioactive cell-wall lipids; exposure of these lipids on the surface of $M$. abscessus may be necessary for intracellular replication in macrophages to occur. To test this hypothesis we examined the ability of $390 \mathrm{~S} \Delta m m p L 4 b$ to replicate in human monocyte-derived macrophages (MDMs). The deletion (a)

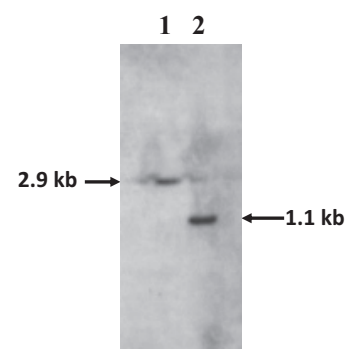

(b)

(1)

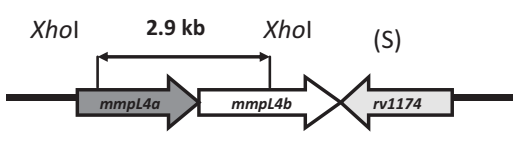

(2)

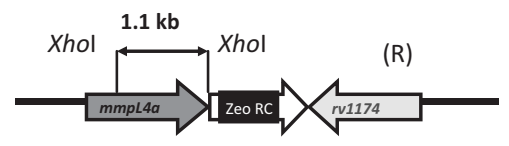

Fig. 2. (a) Southern blot analysis of the $m m p L 4 b$ locus in $M$. abscessus 390 S wildtype (lane 1) and the allelic-exchange mutant (lane 2) using the upstream part of $m m p L 4 b$ as a probe. (b) Schematic representation of the wild-type mmpSL4 operon in $M$. abscessus 3905 (1) and in the allelic-exchange mutant (2). The predicted phenotype is indicated in parentheses: $\mathrm{S}$, smooth; $\mathrm{R}$, rough. 


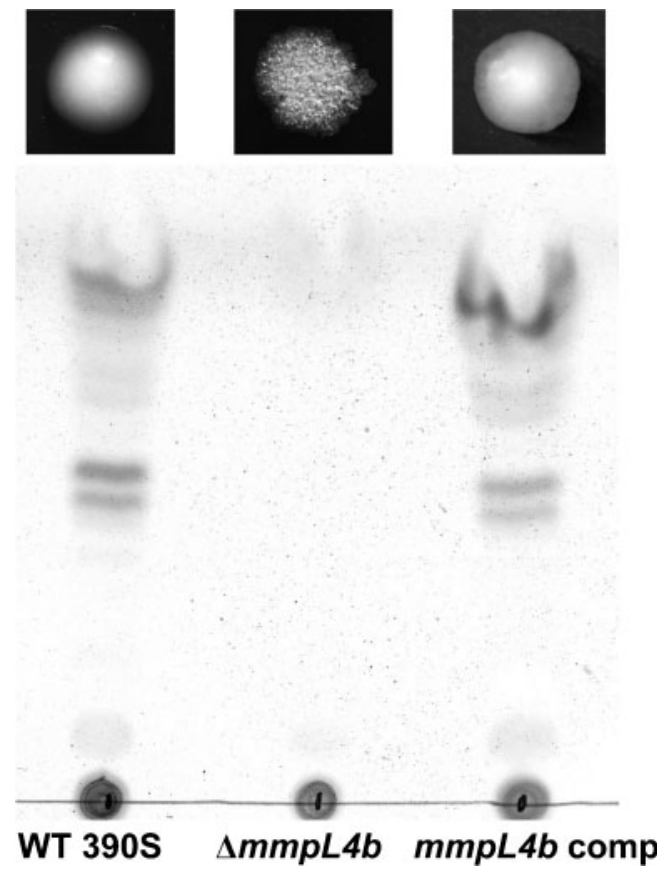

Fig. 3. Characterization of $390 S \Delta m m p L 4 b$ and the complemented strain. Photographs of 3-day-old $M$. abscessus colonies grown at $37^{\circ} \mathrm{C}$ at the top of figure demonstrate the smooth colony phenotype of wild-type strain $390 \mathrm{~S}$ (WT), the rough colony morphology of $m m p L 4 b$ deletion mutant $(\Delta m m p L 4 b)$ and restoration of the smooth phenotype in complemented mutant $(\Delta m m p L 4 b$ comp). Corresponding TLC of extracted lipids (with detection by 1-naphthol) demonstrates characteristic $M$. abscessus GPL species which are absent in the $m m p L 4 b$ deletion mutant.

mutant replicated over 100-fold in MDMs while wild-type $390 \mathrm{~S}$ exhibited minimal growth (Fig. 6a). Infection of MDM monolayers with $390 \mathrm{~S} \Delta m m p L 4 b$ was accompanied by extensive macrophage aggregation beginning at $24 \mathrm{~h}$, accompanied by progressive destruction of monolayers (Fig. 6b). Formation of infected cellular aggregates has been previously reported during MDM monolayer infection with $M$. abscessus rough variants, as well as with virulent M. tuberculosis (Byrd, 1997; Byrd \& Lyons, 1999). The complemented strain did not differ in terms of replication over $24 \mathrm{~h}$ compared to wild-type; however, by $48 \mathrm{~h}$ intracellular replication had begun to occur (Fig. 6a). This was likely due to loss of the plasmid containing $m m p L 4 b$ during the course of the experiment. This is supported by our observation that the percentage of $M$. abscessus rough variant c.f.u. recovered from macrophage lysates infected with the complemented mutant increased dramatically at $48 \mathrm{~h}(9 \%)$ and $72 \mathrm{~h}(37 \%)$, compared to 0 and $24 \mathrm{~h}(0 \%)$. This was accompanied by formation of MDM aggregates beginning at $48 \mathrm{~h}$ (Fig. 6b), although not nearly as extensive as that seen in MDM monolayers infected with $390 \mathrm{~S} \Delta m m p L 4 b$. To ensure that there was no cross-contamination of our tissue culture experiments with M. abscessus wild-type rough variants, which we also use in (a)

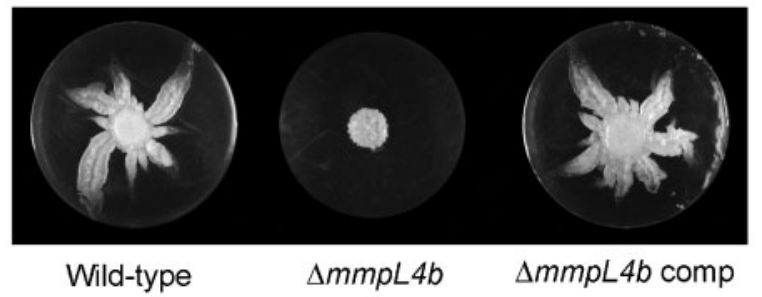

(b)

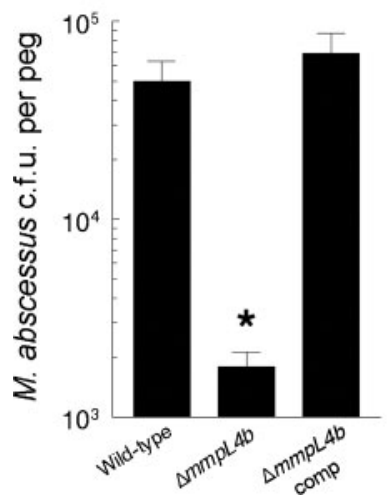

(c)

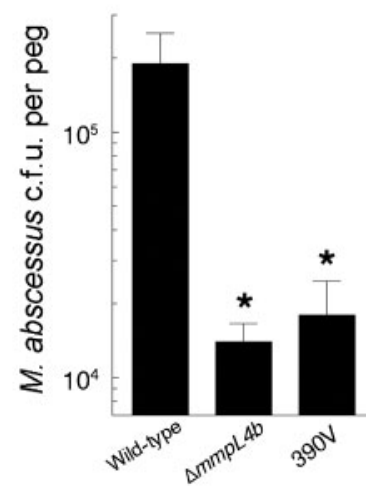

Fig. 4. Loss of colonization capability by $M$. abscessus 3905 $\Delta m m p L 4 b$. (a) M. abscessus $390 S \Delta m m p L 4 b$ has lost sliding motility capability. $390 \mathrm{~S}$ wild-type, $390 \mathrm{~S} \mathrm{mpL} 4 \mathrm{~b}$ deletion mutant ( $\Delta m m p L 4 b)$ and the complemented mutant ( $\Delta m m p L 4 b$ comp) were plated on motility agar and sliding motility was compared at 7 days. (b) M. abscessus 390S $\Delta m m p L 4 b$ has lost biofilm-forming capability. Strains $390 S$ wild-type, $\Delta m m p L 4 b$ and $\Delta m m p L 4 b$ comp were assessed for their ability to form biofilm in a Calgary biofilm device, as described in Methods; c.f.u. in supernates recovered from the peg inserts were determined at $72 \mathrm{~h}$. Data are means \pm SEM of two experiments, each done in triplicate. *390S $\Delta m m p L 4 b$ versus $390 S$ wild-type and $390 \mathrm{~S} \Delta m m p L 4 b$ comp; $P<0.01$, t-test. (c) $M$. abscessus $390 \mathrm{~S} \Delta m m p L 4 b$ biofilm capability is comparable to that of the $390 \mathrm{~V}$ variant. The biofilmforming capability of $390 S \Delta m m p L 4 b$ was compared to that of $390 S$ wild-type and the naturally occurring $390 \mathrm{~V}$ variant, using the assay as in (b). Data are means \pm SD of duplicate determinations. *390S $\triangle m m p L 4 b$ and $390 \mathrm{~V}$ versus $390 \mathrm{~S}$ wild-type; $P<0.05$, t-test.

our laboratory, we plated c.f.u. at all time points on $7 \mathrm{H} 11$ agar with and without zeocin. Whereas c.f.u. from the wells infected with $390 \mathrm{~S} \Delta m m p L 4 b$ or complemented $390 \mathrm{~S}$ $\Delta m m p L 4 b$ had similar numbers of c.f.u. on both plates at all time points, the $390 \mathrm{~S}$ wild-type grew only on the $7 \mathrm{H} 11$ plates without zeocin, confirming the origin of the $M$. abscessus variants throughout the course of the infection experiment. In addition, $390 \mathrm{~S} \Delta m m p L 4 b$ multiplied to a similar extent in MDM monolayers as the comparator strain 390V (Fig. 6c). Our assay is performed by washing MDM monolayers extensively after initial infection to remove extracellular bacteria so as to assess intracellular growth. We have previously found no difference in growth 
(a)

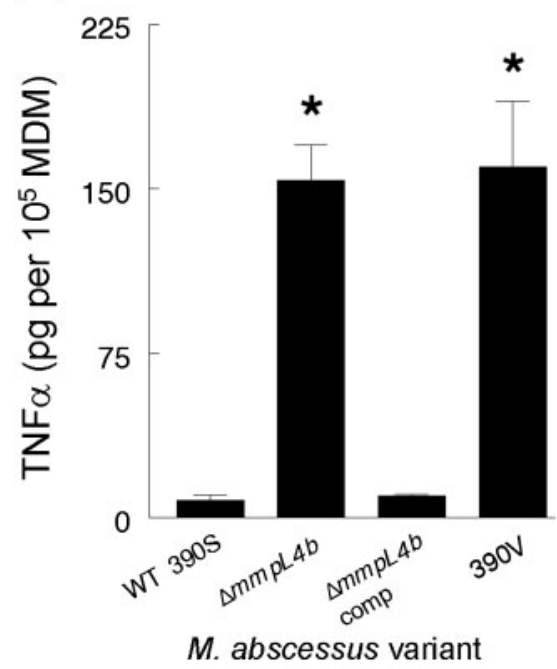

(b)

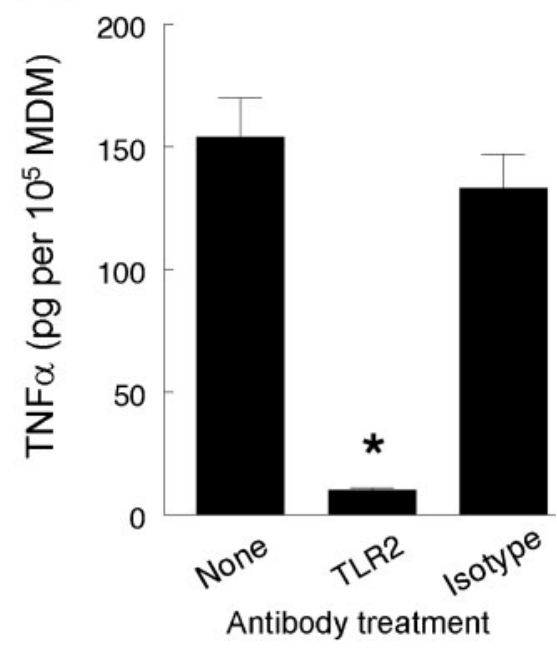

Fig. 5. (a) M. abscessus $390 S \Delta m m p L 4 b$ has acquired the ability to stimulate human MDMs comparable to that induced by the $390 \mathrm{~V}$ variant. Human MDMs were challenged with 390 S wild-type (WT), $390 S \mathrm{mmpL} 4 \mathrm{~b}$ deletion mutant $(\Delta m m p L 4 b)$, the complemented mutant ( $\Delta m m p L 4 b$ comp) and the comparator strain 390V. Culture supernates were collected after $2 \mathrm{~h}$ and assayed by ELISA for TNF $\alpha$. Data are means \pm SEM of two experiments done in duplicate. *390S $\triangle m m p L 4 b$ and $390 \mathrm{~V}$ versus $390 \mathrm{~S}$ wild-type and $390 \mathrm{~S}$ $\Delta m m p L 4 b$ comp; $P<0.01, t$-test. (b) TNF $\alpha$ release from human macrophages in response to $390 \mathrm{~S} \Delta \mathrm{mmpL} 4 \mathrm{~b}$ is blocked by antibody to TLR2. Human MDMs were preincubated with antibody to TLR2 or isotype control antibody and then challenged with $M$. abscessus $390 \mathrm{~S} \Delta m m p L 4 b$. Culture supernates were collected $2 \mathrm{~h}$ after addition of bacteria and assayed by ELISA for TNF $\alpha$. Data are means \pm SEM of two experiments done in duplicate. ${ }^{*} T L R 2$ versus no-antibody and isotype control antibody; $P<0.01$, $t$-test.

of rough and smooth strains in MDM monolayers when comparing extensive washing to extensive washing plus addition of amikacin at the MBC for $6 \mathrm{~h}$ after initial infection (Howard et al., 2006; Greendyke \& Byrd, 2008). Results comparing wild-type $390 \mathrm{~S}$ to $390 \mathrm{~S} \Delta m m p L 4 b$ and $390 \mathrm{~V}$ were identical when amikacin at the MBC was added immediately after infection for $6 \mathrm{~h}$ to ensure killing of extracellular bacteria (Fig. 6d). Thus, these results indicate that a single genetic change associated with loss of an outermost bacterial cell-wall lipid (GPL) is sufficient to convert $M$. abcessus to a phenotype able to replicate within human macrophages.

\section{DISCUSSION}

In this study we have demonstrated loss of GPL expression by the M. abscessus 390 S variant through deletion of the $m m p L 4 b$ gene, which codes for a membrane protein that is critical for GPL expression. To our knowledge, our study is the first to demonstrate that loss of a molecule at the outermost surface of the cell wall converts a bacterium to an intracellular replicative phenotype. In contrast to our findings, other studies have demonstrated that mycobacterial gene deletion mutants generally have reduced pathogenicity and/or ability to replicate in macrophages. For example, deletion of the ompATb gene of $M$. tuberculosis, a gene coding for a putative porin, leads to reduced intracellular replication in macrophages, possibly as a result of diminished ability to respond to reduced phagosomal pH (Raynaud et al., 2002). In addition, using a genetic approach, we confirm the results of our prior study, which was the first to provide evidence that a cell-wall lipid (GPL) can mask underlying immunostimulatory cell-wall lipids (Rhoades et al., 2009).

The explanation for our observations relates to the biological inertness of M. abscessus GPL and the concomitant masking of bioactive cell-wall molecules by GPL in the $M$. abscessus cell wall. It has been demonstrated that GPLs are localized to the outermost portion of the mycobacterial cell envelope of NTM (Barrow et al., 1980), and are responsible for the smooth phenotype which occurs in species of NTM where there are smooth/ rough colony variants (Eckstein et al., 2000; Howard et al., 2006). The GPL molecule consists of a tripeptide-amino alcohol core with an amide-linked long-chain fatty acid. This lipopeptide core is substituted with 6-deoxytalose and $O$-methylated rhamnose to generate the non-specific core GPLs (nsGPLs) (Brennan \& Goren, 1979; Ripoll et al., 2007). This is the form of GPL present in the M. abscessus cell wall. We have previously demonstrated that nsGPL of M. abscessus is not strongly immunogenic (Rhoades et al., 2009). While the nsGPLs of $M$. abscessus, $M$. chelonae and M. smegmatis are structurally identical (Ripoll et al., 2007), the variable haptenic oligosaccharide at 6-deoxytalose present in $M$. avium-intracellulare GPL forms the basis for serotyping $M$. avium-intracellulare strains and is felt to bestow immunogenic properties on serovar-specific GPLs (ssGPLs) (Brennan \& Nikaido, 1995). It has been reported that ssGPLs from smooth variants of M. avium-intracellulare serovars 1 and 2 stimulate macrophage $\mathrm{TNF} \alpha$ release 
(a)

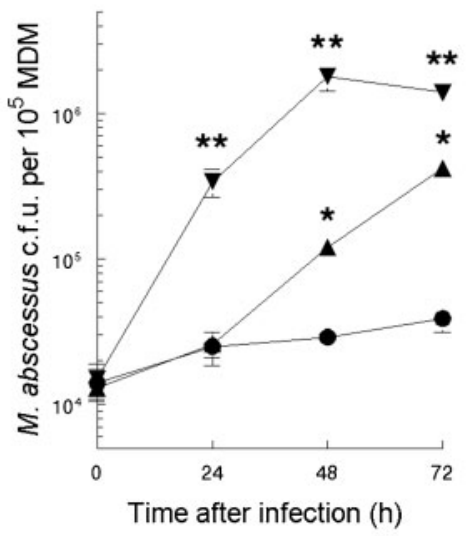

(b)

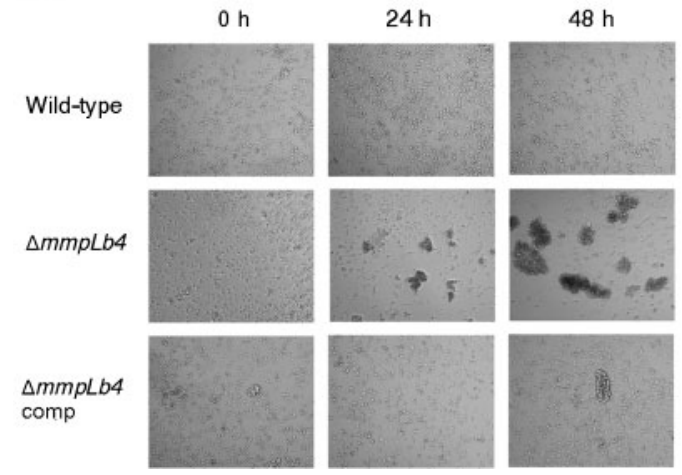

(c)

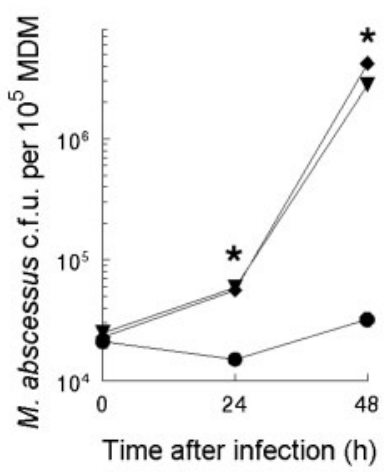

(d)

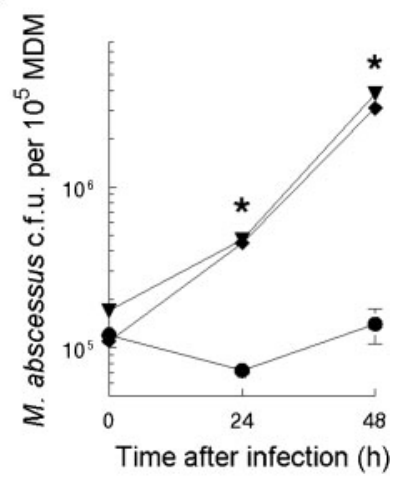

Fig. 6. $M$. abscessus $390 S \Delta m m p L 4 b$ has acquired the ability to replicate in human macrophages. (a) Growth of $M$. abscessus $390 S \Delta m m p L 4 b(\boldsymbol{\nabla})$ compared to $390 S$ wild-type $(\boldsymbol{\bullet})$ and complemented $390 \mathrm{~S} \triangle \mathrm{mmpL} 4 b(\mathbf{\Delta})$. Bacterial c.f.u. in MDM monolayers were determined at the indicated intervals and corrected to c.f.u. per $10^{5} \mathrm{MDM}$. Monolayers were $>98 \%$ viable at time 0 immediately after infection as assessed by trypan blue exclusion. Data are means \pm SEM of two experiments done in duplicate. ${ }^{* *} \Delta m m p L 4 b$ versus wild-type and complemented $\Delta m m p L 4 b ; P<0.01, t$-test. ${ }^{*}$ Complemented $\Delta m m p L 4 b$ versus wild-type; $P<0.01, t$-test. (b) Progression of macrophage infection. Replicate MDM monolayers were fixed and photographed at 0 (immediately after infection), 24 and $48 \mathrm{~h}$ to monitor the effect of infection with the $M$. abscessus variants. Original image taken at $\times 100$ magnification. (c) Growth of $M$. abscessus $390 S \Delta m m p L 4 b(\boldsymbol{\nabla})$ compared to $390 S$ wild-type ( $)$ and the $390 \mathrm{~V}$ comparator strain ( $)$. Bacterial c.f.u. in MDM monolayers were determined at the indicated intervals and corrected to c.f.u. per $10^{5}$ MDM. Monolayers were $>98 \%$ viable at time 0 immediately after infection as assessed by trypan blue exclusion. Data are means \pm SD of duplicate determinations. ${ }^{*} 390 \mathrm{~S} \Delta \mathrm{mmpL} 4 \mathrm{~b}$ and $390 \mathrm{~V}$ versus $390 \mathrm{~S}$ wild-type; $P<0.05, t$-test. (d) Growth of $M$. abscessus 390S $\triangle m m p L 4 b(\boldsymbol{\nabla})$ compared to $390 \mathrm{~S}$ wild-type $(\bullet)$ and the $390 \mathrm{~V}$ comparator strain $(\boldsymbol{})$ with addition of amikacin immediately after infection. The experiment was performed as in (c) except that amikacin at a concentration of $64 \mu \mathrm{g}$ $\mathrm{ml}^{-1}$ (MBC) was added for $6 \mathrm{~h}$ immediately after infection prior to plating c.f.u. at time 0 . Monolayers were $>98 \%$ viable at time 0 immediately after infection as assessed by trypan blue exclusion. Data are means \pm SD of duplicate determinations. ${ }^{*} 390 S$ $\Delta m m p L 4 b$ and $390 \mathrm{~V}$ versus $390 \mathrm{~S}$ wild-type; $P<0.05, t$-test.

via TLR2, while nsGPLs do not (Sweet \& Schorey, 2006). Thus, in contrast to M. avium-intracellulare ssGPL, $M$. abscessus nsGPL is a relatively inert molecule in terms of its ability to stimulate the host innate immune response. However, in addition to nsGPLs, M. abscessus possesses mannose-containing bioactive cell-wall lipids (Rhoades et al., 2009). We have previously demonstrated the presence of one of these classes of molecules known as the phosphatidyl-myo-inositol mannosides (PIMs) in the M. abscessus cell wall (Rhoades et al., 2009). PIMs isolated from $M$. tuberculosis are macrophage TLR2 agonists
(Krutzik \& Modlin, 2004) and are also involved in intracellular trafficking of this pathogen (Chua et al., 2004). We have also demonstrated that identical $M$. abscessus PIMs are present in the 390R and 390V rough variants, and in the $390 \mathrm{~S}$ smooth variant used in this study. Although 390R and 390V stimulate human macrophage TLR2, 390S does not. However, when GPL is selectively removed from the 390 S surface this variant gains the ability to stimulate TLR2. Furthermore, PIMs isolated from all three variants and coated onto polystyrene beads are able to stimulate human macrophage TLR2. Finally, mannose- 
binding lectins bind avidly to the surface of the 390R and $390 \mathrm{~V}$ variants, but not 390S, suggesting that the PIMs found in this variant are masked by GPL in the outermost cell wall (Rhoades et al., 2009). In addition to PIMs, other biosynthetically related mannosylated lipoglycoconjugates such as lipomannan (LM) and mannose-capped lipoarabinomannan (LAM) play important roles in M. tuberculosis pathogenesis (Schlesinger et al., 1994; Torrelles et al., 2006). M. abscessus possesses several orthologues (MAB_1991c, MAB_2689, MAB_0205c) of genes identified as being directly or indirectly involved in synthesis of these glycolipids (Kaur et al., 2007; Scherman et al., 2009; Driessen et al., 2010); it is thus likely that these molecules are also present in M. abscessus.

Since our previous studies utilized naturally occurring $M$. abscessus variants, it could be argued that the spontaneous changes in GPL expression associated with rough/smooth phenotypic variation are accompanied by other changes which affect biofilm forming capability, sliding motility, immunostimulatory activity and the ability to replicate in macrophages. In this study we conclusively demonstrate that deletion of the gene mmpL4b, which is essential for GPL synthesis and transport to the cell surface, is sufficient for M. abscessus $390 \mathrm{~S}$ to acquire an invasive, immunostimulatory phenotype.

Our results support emerging clinical data which indicate that the presence or absence of GPL is associated with human pathogenic potential of $M$. abscessus. The rough colony phenotype has been associated with fatal disseminated infection (Sanguinetti et al., 2001). Epidemiological data indicate that the rough $M$. abscessus colony phenotype is the predominant form isolated from patients with clinical lung disease and/or chronic colonization, whereas the smooth form is isolated from those who are transiently colonized without symptoms and/or from patients with wound infection secondary to contact with contaminated environmental sources (Jönsson et al., 2007). In addition, a recent clinical report documented that deterioration of lung function in a M. abscessus-infected patient was associated with conversion of sputum isolates from the smooth phenotype to an isogenic rough phenotype (Catherinot et al., 2009).

Analysis of the recently published M. abscessus genome has demonstrated a number of genes which are shared in common with two of the pathogens most frequently isolated from the lungs of cystic fibrosis patients Pseudomonas aeruginosa and Burkholderia cepacia (Ripoll et al., 2009). It is postulated that these genes were acquired from distantly related environmental bacteria via horizontal gene transfer, and there is evidence that the proteins encoded by these genes facilitate colonization of the respiratory tract of cystic fibrosis patients (Ripoll et al., 2009). These shared genes may thus facilitate colonization of the respiratory tract by $M$. abscessus GPL-expressing smooth variants in patients with cystic fibrosis by promoting extracellular survival in biofilms. As we have noted, rough, non-GPL-expressing $M$. abscessus variants are capable of invading and replicating in macrophages (Byrd \& Lyons, 1999; Howard et al., 2006; Greendyke \& Byrd, 2008) and respiratory epithelial cells (unpublished observations). Other identified genes in the M. abscessus genome have homology to genes known to play a role in the virulence of the intracellular pathogen $M$. tuberculosis (Ripoll et al., 2009). It is likely that expression of these genes by $M$. abscessus rough variants promotes intracellular survival in macrophages and facilitates invasion into the lung parenchyma. Thus, with recently identified genes facilitating both extra- and intracellular survival, $M$. abscessus is uniquely adapted to survive both in the lung airways and within host cells in the lung parenchyma. The results of our study indicate that the presence or absence of GPL may be the critical determinant in the outcome of $M$. abscessus lung infection.

\section{ACKNOWLEDGEMENTS}

Financial support: Department of Veterans Affairs, a grant from the Cystic Fibrosis Foundation (T.F.B.), and INSERM (J.-M.R.). We thank Halima Medjahed for providing us with the replicative mycobacterial vector pNBV1. Images in this paper were generated in the University of New Mexico \& Cancer Center Fluorescence Microscopy Shared Resource, funded as detailed on the University website (http://hsc.unm.edu/crtc/microscopy/).

\section{REFERENCES}

Barrow, W. W., Ullom, B. P. \& Brennan, P. J. (1980). Peptidoglycolipid nature of the superficial cell wall sheath of smooth-colony-forming mycobacteria. J Bacteriol 144, 814-822.

Brennan, P. J. \& Goren, M. D. (1979). Structural studies on the typespecific antigens and lipids of the Mycobacterium avium-Mycobacterium intracellulare-Mycobacterium scrofulaceum complex. J Biol Chem 254, 4205-4211.

Brennan, P. J. \& Nikaido, H. (1995). The envelope of mycobacteria. Annu Rev Biochem 64, 29-63.

Byrd, T. F. (1997). Tumor necrosis factor alpha (TNF $\alpha)$ promotes growth of virulent $M$. tuberculosis in human monocytes: ironmediated growth suppression is correlated with decreased release of TNF $\alpha$ from iron-treated, infected monocytes. J Clin Invest 99, 25182529.

Byrd, T. F. \& Horwitz, M. A. (1989). Interferon gamma-activated human monocytes downregulate transferrin receptors and inhibit the intracellular multiplication of Legionella pneumophila by limiting the availability of iron. J Clin Invest 83, 1457-1465.

Byrd, T. F. \& Lyons, C. R. (1999). Preliminary characterization of a Mycobacterium abscessus mutant in human and murine models of infection. Infect Immun 67, 4700-4707.

Catherinot, E., Roux, A. L., Macheras, E., Hubert, D., Matmar, M., Dannhoffer, L., Chinet, T., Morand, P., Poyart, C. \& other authors (2009). Acute respiratory failure involving an $\mathrm{R}$ variant of Mycobacterium abscessus. J Clin Microbiol 47, 271-274.

Ceri, H., Olson, M. E., Stremick, C., Read, R. R., Morck, D. \& Buret, A. (1999). The Calgary Biofilm Device: new technology for rapid determination of antibiotic susceptibilities of bacterial biofilms. J Clin Microbiol 37, 1771-1776. 
Chua, J., Vergne, I., Master, S. \& Deretic, V. (2004). A tale of two lipids: Mycobacterium tuberculosis phagosome maturation arrest. Curr Opin Microbiol 7, 71-77.

Cullen, A. R., Cannon, C. L., Mark, E. J. \& Colin, A. A. (2000). Mycobacterium abscessus infection in cystic fibrosis. Colonization or infection? Am J Respir Crit Care Med 161, 641-645.

Daley, C. L. \& Griffith, D. E. (2002). Pulmonary disease caused by rapidly growing mycobacteria. Clin Chest Med 23, 623-632, vii.

De Groote, M. A. \& Huitt, G. (2006). Infections due to rapidly growing mycobacteria. Clin Infect Dis 42, 1756-1763.

Driessen, N. N., Stoop, E. J. M., Ummels, R., Gurcha, S. S., Mishra, A. K., Larrouy-Maumus, G., Nigou, J., Gilleron, M., Puzo, G. \& other authors (2010). Mycobacterium marinum MMAR_2380, a predicted transmembrane acyltransferase, is essential for the presence of the mannose cap on lipoarabinomannan. Microbiology 156, 3492-3502.

Eckstein, T. M., Inamine, J. M., Lambert, M. L. \& Belisle, J. T. (2000). A genetic mechanism for deletion of the ser2 gene cluster and formation of rough morphological variants of Mycobacterium avium. J Bacteriol 182, 6177-6182.

Fauroux, B., Delaisi, B., Clément, A., Saizou, C., Moissenet, D., Truffot-Pernot, C., Tournier, G. \& Vu Thien, H. (1997). Mycobacterial lung disease in cystic fibrosis: a prospective study. Pediatr Infect Dis $J$ 16, 354-358.

Greendyke, R. \& Byrd, T. F. (2008). Differential antibiotic susceptibility of Mycobacterium abscessus variants in biofilms and macrophages compared to that of planktonic bacteria. Antimicrob Agents Chemother 52, 2019-2026.

Griffith, D. E., Girard, W. M. \& Wallace, R. J., Jr (1993). Clinical features of pulmonary disease caused by rapidly growing mycobacteria. An analysis of 154 patients. Am Rev Respir Dis 147, 12711278.

Griffith, D. E., Aksamit, T., Brown-Elliott, B. A., Catanzaro, A., Daley, C., Gordin, F., Holland, S. M., Horsburgh, R., Huitt, G. \& other authors (2007). An official ATS/IDSA statement: diagnosis, treatment, and prevention of nontuberculous mycobacterial diseases. Am J Respir Crit Care Med 175, 367-416.

Howard, S. T. \& Byrd, T. F. (2000). The rapidly growing mycobacteria: saprophytes and parasites. Microbes Infect 2, 1845-1853.

Howard, N. S., Gomez, J. E., Ko, C. \& Bishai, W. R. (1995). Color selection with a hygromycin-resistance-based Escherichia coli-mycobacterial shuttle vector. Gene 166, 181-182.

Howard, S. T., Byrd, T. F. \& Lyons, C. R. (2002). A polymorphic region in Mycobacterium abscessus contains a novel insertion sequence element. Microbiology 148, 2987-2996.

Howard, S. T., Rhoades, E., Recht, J., Pang, X., Alsup, A., Kolter, R., Lyons, C. R. \& Byrd, T. F. (2006). Spontaneous reversion of Mycobacterium abscessus from a smooth to a rough morphotype is associated with reduced expression of glycopeptidolipid and reacquisition of an invasive phenotype. Microbiology 152, 1581-1590.

Jönsson, B. E., Gilljam, M., Lindblad, A., Ridell, M., Wold, A. E. \& Welinder-Olsson, C. (2007). Molecular epidemiology of Mycobacterium abscessus, with focus on cystic fibrosis. J Clin Microbiol 45, $1497-1504$.

Kaur, D., McNeil, M. R., Khoo, K. H., Chatterjee, D., Crick, D. C., Jackson, M. \& Brennan, P. J. (2007). New insights into the biosynthesis of mycobacterial lipomannan arising from deletion of a conserved gene. J Biol Chem 282, 27133-27140.

Krutzik, S. R. \& Modlin, R. L. (2004). The role of Toll-like receptors in combating mycobacteria. Semin Immunol 16, 35-41.
Medjahed, H. \& Reyrat, J. M. (2009). Construction of Mycobacterium abscessus defined glycopeptidolipid mutants: comparison of genetic tools. Appl Environ Microbiol 75, 1331-1338.

Olivier, K. N., Weber, D. J., Wallace, R. J., Jr, Faiz, A. R., Lee, J. H., Zhang, Y., Brown-Elliot, B. A., Handler, A., Wilson, R. W. \& other authors (2003). Nontuberculous mycobacteria. I: multicenter prevalence study in cystic fibrosis. Am J Respir Crit Care Med 167, 828834.

Petrini, B. (2006). Mycobacterium abscessus: an emerging rapidgrowing potential pathogen. APMIS 114, 319-328.

Raynaud, C., Papavinasasundaram, K. G., Speight, R. A., Springer, B., Sander, P., Böttger, E. C., Colston, M. J. \& Draper, P. (2002). The functions of OmpATb, a pore-forming protein of Mycobacterium tuberculosis. Mol Microbiol 46, 191-201.

Recht, J., Martínez, A., Torello, S. \& Kolter, R. (2000). Genetic analysis of sliding motility in Mycobacterium smegmatis. J Bacteriol 182, 43484351.

Rhoades, E. R., Archambault, A. S., Greendyke, R., Hsu, F., Streeter, C. \& Byrd, T. F. (2009). Mycobacterium abscessus glycopeptidolipids mask underlying cell wall phosphatidyl-myo-inositol mannosides blocking induction of human macrophage TNF-alpha by preventing interaction with TLR2. J Immunol 183, 1997-2007.

Ripoll, F., Deshayes, C., Pasek, S., Laval, F., Beretti, J. L., Biet, F., Risler, J. L., Daffé, M., Etienne, G. \& other authors (2007). Genomics of glycopeptidolipid biosynthesis in Mycobacterium abscessus and $M$. chelonae. BMC Genomics 8, 114.

Ripoll, F., Pasek, S., Schenowitz, C., Dossat, C., Barbe, V., Rottman, M., Macheras, E., Heym, B., Herrmann, J. L. \& other authors (2009). Non mycobacterial virulence genes in the genome of the emerging pathogen Mycobacterium abscessus. PLoS ONE 4, e5660.

Sanguinetti, M., Ardito, F., Fiscarelli, E., La Sorda, M., D'Argenio, P., Ricciotti, G. \& Fadda, G. (2001). Fatal pulmonary infection due to multidrug-resistant Mycobacterium abscessus in a patient with cystic fibrosis. J Clin Microbiol 39, 816-819.

Scherman, H., Kaur, D., Pham, H., Skovierová, H. S., Jackson, M. \& Brennan, P. J. (2009). Identification of a polyprenylphosphomannosyl synthase involved in the synthesis of mycobacterial mannosides. J Bacteriol 191, 6769-6772.

Schlesinger, L. S., Hull, S. R. \& Kaufman, T. M. (1994). Binding of the terminal mannosyl units of lipoarabinomannan from a virulent strain of Mycobacterium tuberculosis to human macrophages. I Immunol 152, 4070-4079.

Sermet-Gaudelus, I., Le Bourgeois, M., Pierre-Audigier, C., Offredo, C., Guillemot, D., Halley, S., Akoua-Koffi, C., Vincent, V., Sivadon-Tardy, V. \& other authors (2003). Mycobacterium abscessus and children with cystic fibrosis. Emerg Infect Dis 9, 1587-1591.

Sherwood, J. T., Mitchell, J. D. \& Pomerantz, M. (2005). Completion pneumonectomy for chronic mycobacterial disease. J Thorac Cardiovasc Surg 129, 1258-1265.

Sweet, L. \& Schorey, J. S. (2006). Glycopeptidolipids from Mycobacterium avium promote macrophage activation in a TLR2and MyD88-dependent manner. J Leukoc Biol 80, 415-423.

Torrelles, J. B., Azad, A. K. \& Schlesinger, L. S. (2006). Fine discrimination in the recognition of individual species of phosphatidyl-myo-inositol mannosides from Mycobacterium tuberculosis by C-type lectin pattern recognition receptors. J Immunol 177, 1805-1816.

Edited by: M. Daffé 